\title{
Efficacy of Initial Basal-Supported Oral Therapy with Sitagliptin in Untreated Type 2 Diabetes
}

Keiji Yoshioka

To view enhanced content go to www.diabetestherapy-open.com

Received: August 14, 2013 / Published online: October 15, 2013

(c) The Author(s) 2013. This article is published with open access at Springerlink.com

\section{ABSTRACT}

Introduction: The present study assessed the efficacy of initial basal-supported oral therapy (BOT) with sitagliptin for achievement of glycemic control and subsequent switching from BOT to sitagliptin-based oral therapy.

Methods: Nineteen recently diagnosed type 2 diabetic patients who had received no antidiabetic medication in the previous 2 years were sequentially examined for the 24-week study. Patients were initially treated with a combination of insulin glargine and sitagliptin. Sitagliptin was initiated and maintained at a dose of $50 \mathrm{mg} /$ day, and insulin glargine was started at a dose of $4 \mathrm{U}$ at bedtime and adjusted if needed.

K. Yoshioka $(\bowtie)$

Yoshioka Diabetes Clinic, 4F, The Tower, Bunroku

Hills, Moriguchi Midsite, 11-2, Moriguchi,

Osaka 570-0028, Japan

e-mail: kyoshioka.clin@pearl.ocn.ne.jp

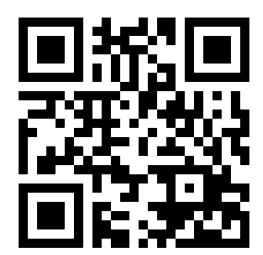

Enhanced content for this article is

available on the journal web site:

www.diabetestherapy-open.com
Results: During the 24-week treatment period, 12 patients (63\%) achieved HbA1c levels $<7 \%$ (mean BOT duration $13.7 \pm 5.6$ weeks) and switched from BOT to sitagliptin monotherapy or in combination with metformin (achievers). The remaining seven patients (37\%) failed to achieve HbA1c levels $<7 \%$ (non-achievers) and continued on BOT. Both FPG and HbA1c in achievers significantly dropped at 4, 8, 12 and 24 weeks from baseline, while those in non-achievers significantly decreased at 12 and 24 weeks from baseline, but failed to reach target glycemic control. There were statistically significant differences in FPG at 4, 8, 12 and 24 weeks and in HbA1c at 8,12 and 24 weeks between achievers and non-achievers. Body weight and BMI in achievers were significantly reduced at 12 and 24 weeks, but those in non-achievers did not change significantly. Dosage of concomitant insulin during BOT was significantly lower in achievers compared to non-achievers. Nonachievers had a similar CPI, a measure of insulin secretion capacity, to achievers, but significantly showed an insulin resistance index (value of 20/[fasting $\mathrm{CPR} \times \mathrm{FPG}]$ ), in comparison to achievers. 
Conclusion: Initiating BOT with sitagliptin followed by sitagliptin-based oral therapy is a useful option in untreated and poorly controlled patients with type 2 diabetes.

Keywords: Basal-supported oral therapy (BOT); Sitagliptin; Type 2 diabetes

\section{INTRODUCTION}

Short-term intensive insulin therapy prescribed early on in the course of type 2 diabetes can improve $\beta$-cell function and insulin resistance by eliminating glucotoxicity and lead to drugfree glycemic remission for up to 2 years $[1,2]$. However, intensive insulin therapy is not generally suitable for outpatients who are often unable to undergo multiple insulin injections; as a result many patients are prescribed oral hypoglycemic agents (OHAs) when they are no longer in glycemic remission. Basal-supported oral therapy (BOT), comprising a basal insulin and OHAs, is an alternative option in this clinical setting [3-5]. However, conventional BOT using sulfonylureas (SUs) mainly controls fasting plasma glucose (FPG) and does not necessarily correct post-prandial glycemia; as a consequence, adequate glycemic control is not always achieved and frequent hypoglycemic episodes may occur [6]. Thus, achieving target glycemic control using conventional BOT is limited.

Dipeptidyl peptidase-4 (DPP-4) inhibitors are used in clinical practice for the treatment of type 2 diabetes $[7,8]$. These agents have an advantage over SUs as they regulate insulin secretion in a glucose-dependent manner [9, 10], thereby minimizing the risk of hypoglycemia [11] and reducing glycemic fluctuations [12, 13]. A few studies evaluating
BOT using DPP-4 inhibitors have demonstrated a glucose-lowering effect when used in conjunction with ongoing insulin therapy in patients with type 2 diabetes [14-16]. The aim of the present study was to evaluate whether starting BOT with sitagliptin, a DPP-4 inhibitor, achieves adequate glycemic control and allows subsequent switching from BOT to sitagliptinbased oral therapy.

\section{MATERIALS AND METHODS}

\section{Patients}

Patients aged between 30 and 70 years were enrolled in the study if they had a new or recent diagnosis of type 2 diabetes, had not been treated with an antidiabetic agent in the previous 2 years, and had poor glycemic control (hemoglobin A1c [HbA1c] $29.0 \%$ ). Patients were excluded if they had type 1 diabetes, chronic liver disease, advanced kidney disease, were taking corticosteroids, or were diagnosed with diabetic ketoacidosis.

\section{Treatment}

Patients were educated in diet therapy [25 kcal $\times$ ideal body weight $(\mathrm{kg})$ per day] by a dietician and instructed to take 12,000 steps per day as exercise, according to the Japanese treatment guide for diabetes [17]. Adherence to diet and exercise therapy was evaluated by the proportion of patients attaining at least $80 \%$ of the instructed levels using self-dietary records and pedometer, respectively.

Patients were treated with a combination of sitagliptin and insulin glargine for 24 weeks. Sitagliptin was initiated and maintained at a dose of $50 \mathrm{mg} /$ day, and insulin glargine was initiated at a dose of $4 \mathrm{U}$ at bedtime and 
adjusted if needed at monthly clinic visit (increased by 1 unit when FPG $>180 \mathrm{mg} / \mathrm{dL}$, and decreased by 1 unit when FPG $<90 \mathrm{mg} / \mathrm{dL}$ ). Patients who achieved an HbA1c of $<7 \%$ during the 24-week treatment period were referred to as achievers and discontinued their basal insulin therapy. They were then maintained on sitagliptin (50 mg/day) monotherapy or on a combination of sitagliptin with metformin (500-1,500 mg/day). Patients who did not achieve an HbA1c of $<7 \%$ (non-achievers) continued on BOT throughout the 24-week treatment period.

\section{Study End Points}

The primary end point was the proportion of patients who achieved an HbA1c $<7.0 \%$ during BOT and discontinued basal insulin during the 24-week treatment period. Secondary end points were changes of FPG and HbA1c from baseline in achievers and non-achievers during the 24-week study period. C-peptide index (CPI), a measure of insulin secretion capacity [18], was calculated at week 12 using the following formula: [100 $\times$ fasting C-peptide immunoreactivity (CPR) (ng/mL)]/[FPG (mg/ $\mathrm{dL})$ ]. In addition, 20/[fasting CPR (mmol/ L) $\times$ FPG $(\mathrm{mmol} / \mathrm{L})]$, a potential index of insulin resistance, was also calculated at week 12 [18]. Lower value of this index indicates being more insulin resistant. HbA1c levels were determined using high-performance liquid chromatography with an automated AH-8280 analyzer (ARKRAY, Kyoto, Japan) at every clinical visit.

\section{Safety Assessment}

Hypoglycemic episodes were counted by documentation of any hypoglycemic episodes and any symptoms derived from hypoglycemia based on patients' reports.

\section{Statistical Analysis}

All statistical analyses were performed using the StatView software package version 5.0 (Abacus Concept, Berkeley, CA). Changes in FPG and HbA1c at 4, 8, 12, and 24 weeks versus baseline were evaluated using ANOVA followed by Bonferroni's test. Unpaired $t$ test was used to compare the parameters between achievers and non-achievers. A $p$ value of $<0.05$ was considered statistically significant. Data are presented as mean ( \pm standard deviation $[S D]$ ).

\section{Ethics Statement}

All procedures followed were in accordance with the ethical standards of the responsible committee on human experimentation (institutional and national) and with the Helsinki Declaration of 1975, as revised in 2000 and 2008. Informed consent was obtained from all patients for being included in the study.

\section{RESULTS}

A total of 22 patients were enrolled in the study and 19 of the participants completed the study protocol. Patient baseline characteristics are summarized in Table 1.

\section{Efficacy}

During the 24-week treatment period, 12 patients (63.2\%) achieved an HbA1c $<7 \%$ using initial BOT (achievers). The mean time to achieve HbA1c $<7.0 \%$ among the achievers was $13.7 \pm 5.6$ weeks. All achievers switched from 
Table 1 Baseline clinical characteristics of the study population

Age (years) $)^{a}$

$48.7 \pm 8.3$

Sex: male/female $(n)$

$16 / 3$

Duration of diabetes (years) ${ }^{a}$

$3.1 \pm 2.3$

Body weight $(\mathrm{kg})$

$78.2 \pm 16.0$

BMI $\left(\mathrm{kg} / \mathrm{m}^{2}\right)$

$26.9 \pm 5.1$

FPG $(\mathrm{mg} / \mathrm{dL})$

$215 \pm 48$

HbAlc (\%) ${ }^{a}$

$11.0 \pm 1.5$

Triglyceride $(\mathrm{mg} / \mathrm{dL})$

$160 \pm 77$

HDL-C $(\mathrm{mg} / \mathrm{dL})^{\mathrm{a}}$

$50 \pm 11$

LDL-C (mg/dL) ${ }^{\mathrm{a}}$

$111 \pm 29$

Hypertension, $n$ (\%)

$6(31.5 \%)$

Dyslipidemia, $n$ (\%)

$9(47.4 \%)$

$B M I$ body mass index, $F P G$ fasting plasma glucose, $L D L$ $C$ low-density lipoprotein cholesterol, $H D L-C$ highdensity lipoprotein cholesterol, $H b A l c$ hemoglobin Alc

${ }^{a}$ Continuous data are means (SD)

BOT to sitagliptin monotherapy or in combination with metformin during the study period. The remaining seven patients (36.8\%) failed to achieve HbA1c levels $<7 \%$ during 24 weeks of treatment (non-achievers).

Rates of adherence to diet and exercise were higher in achievers (diet 75\%; exercise 67\%) than in non-achievers (diet 43\%; exercise 29\%).

Both FPG and HbA1c in achievers significantly dropped at 4, 8, 12 and 24 weeks from baseline, while those in non-achievers significantly decreased at 12 and 24 weeks from baseline, but failed to reach at target glycemic control (Fig. 1). There were statistically significant differences in FPG at 4, 8, 12 and 24 weeks and in $\mathrm{HbA1c}$ at 8, 12 and 24 weeks between achievers and non-achievers.

The achievers experienced a significant reduction in body weight and BMI at 12 and 24 weeks versus baseline, while the nonachievers did not (Table 2). The insulin
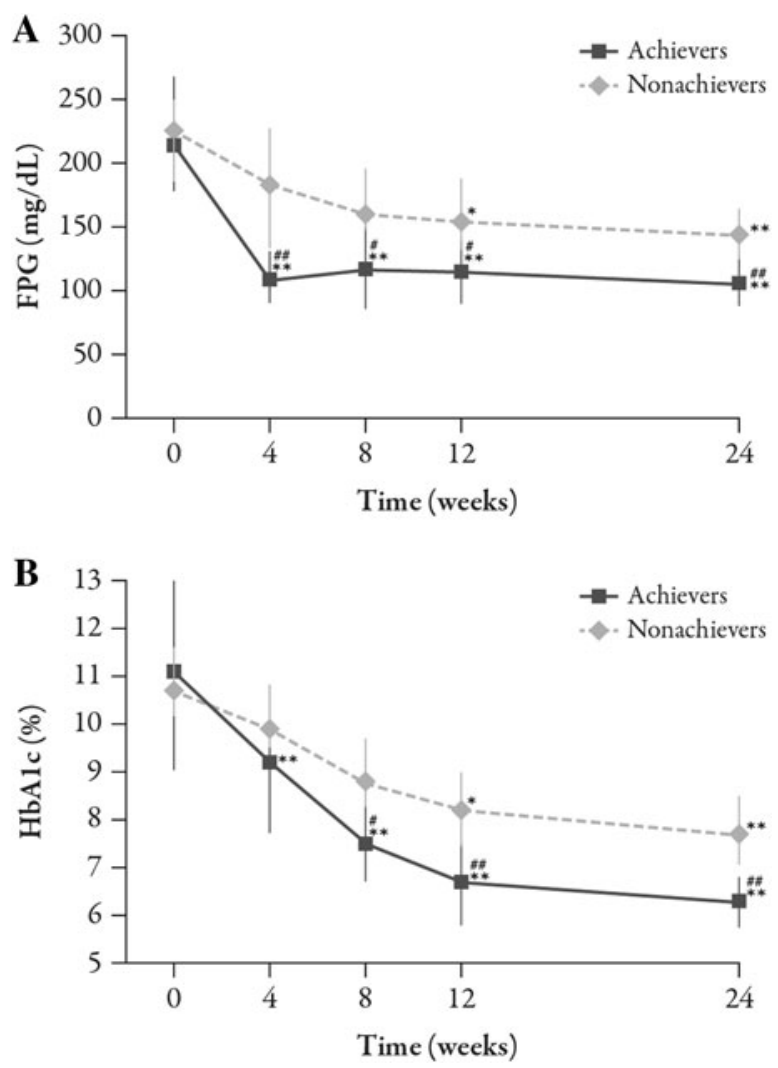

Fig. 1 a Change in fasting plasma glucose (FPG) and b hemoglobin Alc (HbAlc) with time among achievers and non-achievers during the 24-week study period. Achievers: patients who achieved HbAlc of $<7.0 \%$ and switched from BOT to sitagliptin-based oral therapy during the 24-week study period. Non-achievers: patients who failed to achieve $\mathrm{HbAlc}$ of $<7.0 \%$ and continued on BOT during the 24-week study period. ${ }^{*} p<0.05$, ${ }^{* *} p<0.01$ (vs. week 0) ${ }^{\#} p<0.05,{ }^{\# \#} p<0.01$ (achievers vs. non-achievers)

requirement for achievers $(3.8 \pm 0.8 \mathrm{U} /$ day $)$ was significantly lower than that for nonachievers $(7.3 \pm 3.3 \mathrm{U} /$ day $)$ during BOT. Concomitant metformin dosage was significantly lower in achievers $(500 \pm 0 \mathrm{mg}$ / day; $n=2)$ than in non-achievers $(1,050 \pm 450 \mathrm{mg} /$ day; $n=5)$. There was no significant difference in CPI between achievers and non-achievers; however, non-achievers showed a significant insulin resistance index (value of 20/[fasting CPR $\times$ FPG]) compared to achievers. 
Table 2 Comparisons between achievers and nonachievers

\begin{tabular}{|c|c|c|}
\hline & Achievers & $\begin{array}{l}\text { Non- } \\
\text { achievers }\end{array}$ \\
\hline$N$ & 12 & 7 \\
\hline Sex: male/female $(n)$ & $11 / 1$ & $5 / 2$ \\
\hline Age (years) ${ }^{\mathrm{a}}$ & $49.6 \pm 9.6$ & $47.1 \pm 5.8$ \\
\hline $\begin{array}{l}\text { Duration of } \\
\text { diabetes (years) }\end{array}$ & $2.9 \pm 2.0$ & $3.6 \pm 2.7$ \\
\hline Body weight $(\mathrm{kg})$ & $78.1 \pm 13.9$ & $78.3 \pm 20.4$ \\
\hline \multicolumn{3}{|l|}{$\Delta$ Body weight $(\mathrm{kg})^{\mathrm{a}}$} \\
\hline 12 weeks & $-4.2 \pm 3.7^{*}$ & $-0.4 \pm 1.4$ \\
\hline 24 weeks & $-6.6 \pm 5.2^{* *}$ & $0.7 \pm 2.2$ \\
\hline $\operatorname{BMI}\left(\mathrm{kg} / \mathrm{m}^{2}\right)$ & $26.3 \pm 3.8$ & $28.0 \pm 7.0$ \\
\hline \multicolumn{3}{|l|}{$\Delta \mathrm{BMI}\left(\mathrm{kg} / \mathrm{m}^{2}\right)$} \\
\hline 12 weeks & $-1.8 \pm 1.3^{* *}$ & $0.1 \pm 0.9$ \\
\hline 24 weeks & $-1.8 \pm 1.7^{* *}$ & $0.3 \pm 0.9$ \\
\hline $\begin{array}{l}\text { Insulin dose during } \\
\text { BOT (U/day) }\end{array}$ & $3.8 \pm 0.8^{* *}$ & $7.3 \pm 3.3$ \\
\hline CPI at 12 weeks & $1.7 \pm 0.5$ & $1.4 \pm 0.6$ \\
\hline $\begin{array}{l}20 /(\mathrm{FPG} \times \mathrm{CPR}) \\
\text { at } 12 \text { weeks }\end{array}$ & $6.2 \pm 2.1^{*}$ & $3.8 \pm 1.3$ \\
\hline
\end{tabular}

$B O T$ basal-supported oral therapy, CPR C-peptide immunoreactivity, CPI C-peptide index, $F P G$ fasting plasma glucose

${ }^{*} p<0.05,{ }^{* *} p<0.01$ by unpaired $t$ test (achievers vs. nonachievers)

${ }^{a}$ Continuous data are means (SD). $\Delta$ Body weight: change from baseline, $\Delta \mathrm{BMI}$ : change from baseline

\section{Safety}

Two patients in the achiever group and one patient in the non-achiever group experienced hypoglycemia; there were no cases of severe hypoglycemia.

No patients had to discontinue the present protocol because of other side effects.

\section{DISCUSSION}

The current study demonstrated that initiating BOT with sitagliptin successfully improved glycemic control and allowed patients to switch from BOT to sitagliptin monotherapy or combination therapy, maintaining adequate glycemic control during a 24 -week period in untreated patients with type 2 diabetes.

BOT is often prescribed for outpatients because once daily injection is more acceptable than multiple insulin injections. The benefit of BOT in clinical practice has been described previously [3-5]. BOT has traditionally comprised an SU plus a long-acting insulin analog [5]. However, one of the biggest problems associated with conventional BOT is postprandial hyperglycemia while FPG is within normal range. DPP-4 inhibitors, as monotherapy or combination therapy, have several advantages over SUs, since these agents enhance insulin secretion in a glucose-dependent manner and suppress glucagon secretion $[9,10]$.

In the current study, 12 patients (63.2\%) achieved HbA1c $<7 \%$ using initial BOT, and subsequently discontinued basal insulin. These achievers showed a significant decrease in HbA1c at 4, 8, 12 and 24 weeks versus baseline, and versus non-achievers at 8, 12 and 24 weeks. The basal insulin dose was relatively low and the duration of BOT was short to achieve target glycemic control. In contrast, the remaining seven patients $(36.8 \%)$ failed to achieve a target HbA1c $<7 \%$ during the 24-week treatment period, although in common with the achievers they adhered to their instructed insulin injection. It is reported that insulin therapy is usually required in Japanese type 2 diabetic patients with a CPI (insulin secretion capacity) lower than 0.8 [18]. CPI was measured at 12 weeks because 
glucotoxicity might have influenced this index at baseline. In comparison to the achievers, the non-achievers had a similar CPI, but a significantly lower 20/[fasting CPR $\times$ FPG] level, a possible marker of insulin resistance [19] at 12 weeks. Lower value of this index indicates being more insulin resistant. Body weight and BMI in non-achievers were not significantly reduced from baseline, probably because these patients did not strictly adhere to diet and exercise therapy. These results suggest that insulin resistance was not sufficiently improved to allow non-achievers to discontinue basal insulin.

In the Add-on Lantus ${ }^{\circledR}$ to Oral Hypoglycemic Agents (ALOHA) study in insulin-naïve 3,515 Japanese patients starting insulin glargine (mean dose $8.5 \mathrm{U} /$ day) plus OHAs, mainly SUs, $15.5 \%$ of participants achieved HbA1c $<7.0 \%$ at 24 weeks, whereas $84.5 \%$ of participants did not. In such a study, a shorter duration of diabetes ( $<1$ year) and lower HbA1c $(<8.5 \%)$ at baseline were significantly associated with a higher rate of achieving target HbA1c $(<7.0 \%)$ [20]. Previous BOT trials [14-16] with DPP-4 inhibitors added to ongoing insulin therapy in patients with type 2 diabetes have demonstrated a significant glucose-lowering effect; however, the background of previous studies [14-16] where duration of diabetes was over 10 years, daily insulin dose exceeded $15 \mathrm{U} /$ day and concomitant OHAs were frequently used is quite different from the current study. Recently, Harashima et al. [21] have demonstrated in a 52-week study of ongoing BOT with SUs that replacement of basal insulin with sitagliptin was associated with a decrease in HbA1c level $(<7.0 \%)$ in $67.4 \%$ of patients with a mean diabetes duration of 12.1 years, while $32.6 \%$ of subjects with a longer duration of diabetes ( $\sim 18.9$ years) and receiving higher doses of concomitant SUs could not replace basal insulin by sitagliptin. Taken together, the efficacy of BOT in type 2 diabetes patients may be related to several factors of duration of diabetes, pre-treatment or insulin resistance.

This study has some limitations. First, this was a single arm, single center study involving a small sample of patients. It was unable to compare the efficacy and convenience of initial treatments between the current study of BOT with sitagliptin and other therapies by BOT with SU or multiple insulin injection regimen. Second, the study findings cannot be generalized to patients with type 2 diabetes who would not meet the study inclusion criteria, i.e., drug-naïve patients with recently diagnosed, poorly controlled type 2 diabetes. Third, the titration of basal insulin and adherence to diet and exercise may have been insufficient for the non-achievers. On this basis, a large prospective study is needed to validate the findings of the current study.

\section{CONCLUSION}

The current study showed that initial introduction of BOT with sitagliptin was effective for achievement of glycemic control and subsequent switching from BOT to DPP-IV inhibitor-based therapy in recent onset and untreated patients with type 2 diabetes.

\section{ACKNOWLEDGMENTS}

No funding or sponsorship was received for this study or publication of this article. Dr. Yoshioka is the guarantor for this article and takes responsibility for the integrity of the work as a whole.

Conflict of interest. Keiji Yoshioka declares no conflict of interest. 
Compliance with ethics guidelines. All procedures followed were in accordance with the ethical standards of the responsible committee on human experimentation (institutional and national) and with the Helsinki Declaration of 1975, as revised in 2000 and 2008. Informed consent was obtained from all patients for being included in the study.

Open Access. This article is distributed under the terms of the Creative Commons Attribution Noncommercial License which permits any noncommercial use, distribution, and reproduction in any medium, provided the original author(s) and the source are credited.

\section{REFERENCES}

1. Retnakaran R, Drucker DJ. Intensive insulin therapy in newly diagnosed type 2 diabetes. Lancet. 2008;371:1725-6.

2. Chen HS, Wu TE, Jap TS, et al. Beneficial effects of insulin on glycemic control and beta-cell function in newly diagnosed type 2 diabetes with severe hyperglycemia after short-term intensive insulin therapy. Diabetes Care. 2008;31:1927-32.

3. Shreiber RR, Ferlinz K, Haak T. The long-term efficacy of insulin glargine plus oral antidiabetic agents in a 32-month observational study of everyday clinical practice. Diabetes Technol Ther. 2008;10:121-7.

4. Kostev K, Dippel FW. Predictors for initiation of a basal supported oral therapy (BOT) in type 2 diabetic patients under real-life conditions in Germany. Prim Care Diabetes. 2012;6:329-35.

5. Ohtani T, Ito T. Safety and effectiveness of BOT (Basal supported Oral Therapy) using insulin glargine in Japanese patients with type 2 diabetesresults from postmarketing surveillance of insulin glargine (ALOHA study). Shinyaku to Rinsho (New Rem Clin). 2011;60:458-75 (in Japanese).

6. Holman RR, Farmer AJ, Davies MJ, et al.; 4-T Study Group. Three-year efficacy of complex regimens in type 2 diabetes. N Engl J Med. 2009;361:1736-47.

7. Sheen AJ. DDP-IV inhibitors in the management of type 2 diabetes: a critical review of head-to-head trials. Diabetes Metab. 2012;38:89-101.
8. Crepaldi G, Carruba M, Comaschi $M$, et al. Dipeptidyl peptidase 4 (DDP-4) inhibitors and their role in type 2 diabetes management. J Endocrinol Invest. 2007;30:610-4.

9. Ahren B, Holst JJ, Martensson H, Balkan B. Improved glucose tolerance and insulin secretion by inhibition of dipeptidyl peptidase IV in mice. Eur J Pharmacol. 2000;404:239-45.

10. Seino Y, Fukushima M, Yabe D. GIP and GLP-1, two incretin hormones: similarities and differences. J Diabetes Invest. 2010;1:8-23.

11. Iwamoto Y, Kashiwagi A, Yamada N, et al. Efficacy and safety of vildagliptin and voglibose in Japanese patients with type 2 diabetes: a 12-week, randomized, double-blind, active controlled study. Diabetes Obes Metab. 2010;12:700-8.

12. Sakamoto $M$, Nishimura $R$, Irako $T$, et al. Comparison of vildagliptin vs. sitagliptin one daily using continuous glucose monitoring (CGM): crossover pilot study (J-VICTORIA study). Cardiovasc Diabetol. 2012;11:92-8.

13. Yoshioka $\mathrm{K}$, Isotani $\mathrm{H}$, Ohashi $\mathrm{S}$, Imamura $\mathrm{M}$. Efficacy of vildagliptin on glucose fluctuation in Japanese type 2 diabetic patients with ongoing sulfonylurea based oral glycemic agent therapy. Diabetes Metab Syndr. 2013;7:32-4.

14. Vilsbøll T, Rosebstock J, Yki-Järvinen $\mathrm{H}$, et al. Efficacy and safety of sitagliptin when added to insulin therapy in patients with type 2 diabetes. Diabetes Obes Metab. 2010;12:167-77.

15. Fonseca V, Schweizer A, Albrecht D, et al. Addition of vildagliptin to insulin improves glycemic control in type 2 diabetes. Diabetologia. 2007;50:1148-55.

16. Katsuno T, Ikeda H, Ida K, Miyagawa J, Namba M. Add-on therapy with the DPP-4 inhibitor sitagliptin improves glycemic control in insulin-treated Japanese patients with type 2 diabetes mellitus. Endocr J. 2013;60:733-42.

17. Japan Diabetes Society. Dietary and exercise therapy. In: Treatment guide for diabetes 2012. Tokyo: Bunkodo Co., Ltd. 2012;39-45 (in Japanese).

18. Funakoshi S, Fujimoto S, Hamasaki A, et al. Analysis of factors influencing pancreatic beta-cell function in Japanese patients with type 2 diabetes. Association with body mass index and duration of diabetic exposure. Diabetes Res Clin Pract. 2008;82:353-8.

19. Ohkura T, Shiochi H, Fujioka Y, et al. 20/(fasting C-peptide $\times$ fasting plasma glucose) is a simple and effective index of insulin resistance in patients with 
type 2 diabetes mellitus: a preliminary report. Cardiovasc Diabetol. 2013;12:21-8.

20. Kadowaki T, Ohtani T, Odawara M. Baseline predictive factors for glycemic control in Japanese type 2 diabetes patients treated with insulin glargine plus oral antidiabetic drugs:
ALOHA study subanalysis. Diabetol Int. 2013;4: $16-22$.

21. Harashima SI, Tanaka D, Yamane S, et al. Efficacy and safety of switching from basal insulin to sitagliptin in Japanese type 2 diabetes patients. Horm Metab Res. 2013;45:231-8. 